\title{
Postoperative vocal fold dysfunction in covid-19 era: are we still in time for a recovery?
}

\author{
Elena Bonati $i^{1}$ - Elena Giovanna Bignami ${ }^{2} \cdot$ Paolo Del Rio ${ }^{1}$
}

Received: 14 May 2020 / Accepted: 21 July 2020 / Published online: 9 August 2020

(c) Springer Science+Business Media, LLC, part of Springer Nature 2020

\section{To the Editor:}

The novel 2019 coronavirus (COVID-19) is a highly contagious zoonosis produced by SARS-CoV-2, which arose in China and spread all over the world, transmitting from man to man through respiratory secretions. In March 2020, it was defined by the World Health Organization (WHO) as a pandemic, to underline its spread and severity.

Healthcare professionals are one of the categories most at risk of contracting the infection, in particular when their activity involves the direct management of the patient's airways. Among these categories, we can count anesthetists, head and neck surgeons, otolaryngologists, maxillofacial surgeons, ophthalmologists, and dentists. For these reasons, the latest evidence-based recommendations for otolaryngology and head and neck surgery practice suggest that healthcare facilities should prioritize urgent and emergency visits and procedures until this condition stabilizes, ceasing elective care [1].

Nevertheless, oncological surgical activity, although slowed down, did not stop in most hub hospitals. Regarding thyroid cancer, thyroid surgery is complex and the rate of nerve damage is still considerable. Immediate postoperative vocal fold rate is $2-4 \%$ in our case study, and decrease to 1-2\% after 6 months. Postoperative dysphonia can be caused by several factors other than nerve damage, such as tracheal intubation or scarring in the thyroid lodge. It is, therefore, important to identify the cause of vocal cord dysfunction and treat it correctly, at the right time. If an unilateral vocal fold paresis/paralysis is diagnosed, the treatment consist in improving the speech, while, in case of

Elena Bonati

ebonati86@gmail.com

1 General Surgery Unit, Department of Medicine and Surgery, Parma University Hospital, Parma, Italy

2 Unit of Anesthesiology, Department of Medicine and Surgery, Parma University Hospital, Parma, Italy bilateral vocal fold paresis/paralysis, respiratory obstruction also needs to be urgently treated. Fortunately, we have brought the incidence of this last and most dangerous complication to $0 \%$ at our Clinic, since the introduction in 2014 of the routine use of intraoperative neuromonitoring during thyroidectomy.

The latest guidelines published by the American Association of Endocrine Surgeons in March 2020 recommend laryngeal examination in patients with known or suspected new recurrent laryngeal nerve dysfunction after thyroidectomy, for additional evaluation and possible treatment with a speech pathologist. According to the American Academy of Otolaryngology-Head and Neck Surgery, they assert that early referral (2-8 weeks post surgery) to a laryngologist, in combination with early intervention, results in superior voice outcomes, since the ideal time for vocal fold augmentation is $<3$ months after thyroidectomy [2].

A meta-analysis about therapy for vocal fold paresis/ paralysis after thyroidectomy concluded that the timing of therapy for unilateral vocal fold paralysis after thyroidectomy has a significant impact on the effect size, being significantly greater if therapy is performed within 12 months. This may be explained by progressive atrophy of the vocal folds and disappearance of nerve function, so that vocal fold movements cannot be recovered [3].

Patients who underwent thyroid surgery from February 2020 and who had experienced a vocal fold disfunction (VFD) were unable to undergo a laryngoscopy nor, much less, a speech therapy, according to health measures necessary to contain the spread of the virus. This, unfortunately, causes a progressively reduced possibility of recovery, increasing the specific morbidity related to surgery for thyroid cancer in this period. The only indication that we can give to patients is the rest of the voice, to avoid the establishment of compensation mechanisms worsening the clinical picture, waiting to be able to resume the correct treatment. 
Therapeutic diagnostic pathways in the COVID-19 era have become difficult and dangerous logarithms that must consider the need for patient care and the possibility of treatments delay in safety, but also the risk of contagion of the patients themselves and, not least, protection of healthcare personnel. The hospital setup has been significantly changed and much of the economic, structural, and human health resources have been dedicated to the management of the COVID pandemic.

In parallel with the COVID-19 emergency, we are experiencing another health emergency, the one that involves the management of non-COVID-19 patients. Even in the second phase of the pandemic, only urgent health services are provided. A reorganizing effort within the individual healthcare companies is required to guarantee treatment even for non-COVID-19 patients.

The COVID-19 pandemic highlighted the limits and weaknesses of our health system, and now that the correct protocols for the protection of healthcare personnel have been described, all the all healthcare companies should equip their staff with the appropriate materials, such as N95 masks, hair cover, protective coverall, gown, gloves, face shields, goggles, and shoe covers. In the face of higher expenses, this would allow the resumption of activities, minimizing the risk of an increase in the rate of infection.

Moreover, routine health practices must be reconsidered, preferring less invasive techniques, in order to screen patients who need second-level examination. Even if not used yet in our hospital, transcutaneous laryngeal ultrasonography is a valid noninvasive and painless alternative method in the assessment of vocal cords. It has been demonstrated in a recent prospective multicentric study that it has concordance with laryngoscopy in the majority of cases and so it can be a valid alternative as first-line exam for vocal fold examination, pre- and postoperatively.
Finally, the growing use of virtual platforms for the need of social distancing could encourage their application even in healthcare services that can be performed by teleconference, such as speech therapy.

We can assess that COVID-19 pandemic is causing direct morbidity and mortality, and even a related one, due to missed or delayed treatment of multiple non-COVID-19 diseases. The delivery of the health service should be improved and the health system itself must be modernized to adapt to new needs.

\section{Compliance with ethical standards}

Conflict of interest The authors declare that they have no conflict of interest.

Publisher's note Springer Nature remains neutral with regard to jurisdictional claims in published maps and institutional affiliations.

\section{References}

1. L.P. Kowalski, A. Sanabria, J.A. Ridge, W.T. Ng, R. de Bree, A. Rinaldo, R.P. Takes, A. A. Mäkitie, A.L. Carvalho, C.R. Bradford, V. Paleri, D.M. Hartl, V. Vander Poorten, I.J. Nixon, C. Piazza, P.D. Lacy, J.P. Rodrigo, O. Guntinas-Lichius, W.M. Mendenhall, A. D'Cruz, A.W.M. Lee, A. Ferlito, COVID-19 pandemic: effects and evidence-based recommendations for otolaryngology and head and neck surgery practice. Head Neck (2020). https://doi.org/10. 1002/hed.26164.

2. K.N. Patel, L. Yip, C.C. Lubitz, E.G. Grubbs, B.S. Miller, W. Shen, P. Angelos, H. Chen, G.M. Doherty, T.J. Fahey 3rd, E. Kebebew, V.A. Livolsi, N.D. Perrier, J.A. Sipos, J.A. Sosa, D. Steward, R.P. Tufano, C.R. McHenry, S.E. Carty, The American Association of Endocrine Surgeons Guidelines for the definitive surgical management of thyroid disease in adults. Ann. Surg. 271, e21-e93 (2020)

3. X. Chen, P. Wan, Y. Yu, M. Li, Y. Xu, P. Huang, Z. Huang, Types and timing of therapy for vocal fold paresis/paralysis after thyroidectomy: a systematic review and meta-analysis. J. Voice $\mathbf{2 8}$, 799-808 (2014) 